\title{
Gestão de informação em saúde: processo de digitalização de prontuários em uma maternidade de alta complexidade na cidade de São Luís-MA
}

RESUMO | O objetivo é descrever os estágios de adequação do processo de digitalização de prontuários em uma maternidade em São Luís-MA. O início foi em julho de 2018 com a realização de diagnóstico da situação documental, organização de prontuários com o Serviço de Arquivo Médico e instalação do sistema, com o apoio da Secretaria Estadual da Saúde, Instituto Acqua e o setor técnico de informática. A documentação manual deu-se a partir de 1998 até o ano em curso. O processo conta com 3.603 prontuários digitalizados. Os dados referentes há 11 anos estão catalogados para entrega à Secretaria Estadual de Saúde para o arquivo permanente. Em um ano é possível mensurar os principais ganhos como a agilidade, visualização e impressão dos prontuários. A perspectiva é melhor atender as demandas. O projeto é embrionário e um marco na otimização da documentação digital servindo como referência na eficiência administrativa.

Palavras-chaves: Gestão Hospitalar; Maternidade de Alta Complexidade; Serviço de Arquivo Médico.

ABSTRACT | The aim is to describe the stages of adequacy of the process of digitalization of medical records in a maternity hospital in São Luís-MA. The beginning was in July 2018 with the diagnosis of the documentary situation, organization of medical records with the Medical Archive Service and installation of the system, with the support of the State Department of Health, the Acqua Institute and the IT department. Manual documentation took place from 1998 until the current year. The process has 3,603 digitized medical records. The data for 11 years are cataloged for delivery to the State Department of Health for permanent archive. In a year it is possible to measure the main gains such as agility, visualization and printing of medical records. The prospect is better meet the demands. The project is embryonic and a milestone in the optimization of digital documentation serving as a reference in administrative efficiency.

Keywords: Hospital Management; High Complexity Maternity; Medical File Service.

RESUMEN | El objetivo es describir las etapas de adecuación del proceso de digitalización de registros médicos en un hospital de maternidad en São Luís-MA. El comienzo fue en julio de 2018 con el diagnóstico de la situación documental, la organización de registros médicos con el Servicio de Archivo Médico y la instalación del sistema, con el apoyo del Departamento de Salud del Estado, el Instituto Acqua y el departamento de TI. La documentación manual tuvo lugar desde 1998 hasta el año en curso. El proceso tiene 3,603 registros médicos digitalizados. Los datos de 11 años están catalogados para su entrega al Departamento de Salud del Estado para su archivo permanente. En un año es posible medir las principales ganancias, como la agilidad, la visualización y la impresión de registros médicos. La perspectiva es mejor satisfacer las demandas. El proyecto es embrionario y un hito en la optimización de la documentación digital que sirve como referencia en eficiencia administrativa.

Descriptores: Complejidad; Servicio de Archivo Médico; Digitalización de Historias Clínicas.

\section{Maria Margarida de Carvalho Neta}

Gestora Hospitalar e Enfermeira. Gerente Administrativa da Maternidade de Alta Complexidade do Maranhão - MACMA, em São Luís-MA.

\section{Ana Cássia Martins Ribeiro Cruz}

Enfermeira. Adjunta da subseção de Auditoria em Saúde. Esquadrão de SaúdeForça Aérea Brasileira- Alcântara-MA.

\section{Joelma da Silva Raposo}

Enfermeira. Auditora em Saúde/ Secretaria do Estado de Saúde-SES. Presidente Dutra-MA.

Recebido em: 24/07/2019

Aprovado em: 29/07/2019

\section{Wesley Thauann dos Santos Albuquerque}

Bacharel em Ciências Contábeis. Agente Administrativo. Maternidade de Alta Complexidade do Maranhão - MACMA.

\section{INTRODUÇÃO}

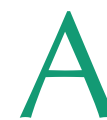
tecnologia da informação configura-se uma ferramenta indispensável à implantação dos processos de reestruturação global, um dos principais fatores de mudanças ocorridas nas várias formas de relações ${ }^{(1)}$. Nesse cenário, as organizações também se encontram no viés do gerenciamento e utilização de tecnologia transformando, de forma constante, seus sistemas de informação. Como enfatizam autores ${ }^{(2)}$, com a disponibilização das tecnologias da informação, as organizações têm utilizado esse recurso para dar apoio ao fornecimento de dados proporcionando acessos dinâmicos e personalizados que facilitam a administração no cotidiano.

Novos sistemas e métodos de informação são utilizados como estratégias visando a ampliação técnica das gerências no seio das diversas formas de negócios. Autores ${ }^{(3: 210)}$ acentuam que "os sistemas de informações, sejam eles assistenciais ou epidemiológicos, compreendidos como instrumento de trabalho na saúde, têm contribuído nas ações administrativas e burocráticas ancoradas em conhecimento técnico-científico". 
A evolução da informática atinge todos os setores da sociedade e, no âmbito da gestão de informação hospitalar, estudo $^{(4)}$ aponta que o uso dos sistemas significa a melhoria de armazenamento e preservação das informações, quer seja na área pública ou privada.

No que se refere à documentação e saúde, destacando o prontuário médico, observa-se autor ${ }^{(5: 19)}$, que cita a Resolução de n. ${ }^{\circ}$ 1.638/2002, Art. $1^{\circ}$ do Conselho Federal de Medicina (CFM) sobre normas técnicas para uso de sistema informatizado, em que define que o prontuário médico é "documento único constituído de um conjunto de informações [...] sobre a saúde do paciente e a assistência prestada. Possibilita a comunicação entre membros da equipe multiprofissional e a continuidade da assistência prestada ao indivíduo". Quanto ao volume crescente de documentos armazenados nos diversos estabelecimentos de saúde, esclarece ainda que os sistemas de armazenamentos de dados passaram a subsidiar as informações sobre a manutenção da saúde de pacientes, favorecendo o compartilhamento das mesmas entre os profissionais da área.

Nesse âmbito, autor ${ }^{(6: 9)}$ explica que "toda documentação criada nas diversas fases de atendimento e atividades exercidas pela instituição deve ser preservada, necessitando de ser concentrada em locais adequados". No processo de armazenamento eletrônico, diversas etapas são imprescindíveis, como a classificação da massa documental, organização para formação dos arquivos e digitalização, visando facilitar a busca, o armazenamento e dar celeridade no acesso à documentação, sendo necessária sua preservação ${ }^{(7)}$.

Todos os aspectos mencionados dão base à apresentação do processo de digitalização que ocorre em uma maternidade na cidade de São Luís-MA, com o intuito margeado na Gestão em Saúde, beneficiando, assim, o andamento do uso dinâmico das informações de prontuários médicos, cuja referência é o acolhimento e atendimento humanizado voltado para pacientes obstétricas, na rede pública de saúde do Maranhão.

Assim, esse artigo apresenta a experiência vinculada à preocupação: Como preservar toda a documentação de registro do atendimento aos pacientes que se avoluma em 21 anos desde o início das atividades da maternidade? Nesse aspecto, referente ao processo eletrônico de preservação de documentação, o objetivo é descrever os estágios em que se encontra a digitalização de prontuários na referida maternidade.

\section{METODOLOGIA}

O presente artigo de caráter exploratório e descritivo trata da digitalização de prontuários de pacientes como recurso de gestão hospitalar para a Maternidade de Alta Complexidade do Maranhão (MACMA). Contemplou, inicialmente, o encontro dos pressupostos que embasaram teoricamente este artigo, através de publicações que dispõem de dados em revistas, artigos eletrônicos e periódicos.

Apoia-se, seguidamente, nas determinações e normas estabelecidas pelos órgãos competentes cujas disposições servem de pilar para seu desenvolvimento. Assim, parte da Portaria do Ministério da Saúde n. ${ }^{\circ}$ 900, de 31 de março de 2017, que dispõe sobre a implantação e o funcionamento do Sistema Eletrônico de Informações - SEI ${ }^{(8)}$. Outras determinações são evidenciadas pelo então Governador do Estado do Maranhão, Flávio Dino, através do Decreto n. ${ }^{\circ} 32.607$, de 25 de janeiro de 2017, que "dispõe sobre os documentos de arquivo e gestão". No uso de suas atribuições: "considerando estabelecer um programa de gestão documental que integre as fases corrente, intermediária e permanente, pelas quais tramitam os documentos de arqui$\operatorname{vos}^{\prime \prime(8: 5)}$. Destaca-se também a Resolução n. ${ }^{\circ}$ 1.821/2007 do Conselho Federal De Medicina - CFM (publicada no D.O.U. de 23 nov. 2007, Seção I, pg. 252), que aprova as normas técnicas concernentes à digitalização e uso dos sistemas informatizados para a guarda e manuseio dos documentos dos prontuários dos pacientes, autorizando a eliminação do papel e a troca de informação identificada em saúde $^{(9)}$.

A partir da viabilização das propostas para a digitalização de prontuários da maternidade, foco deste estudo, foi elaborado planejamento das etapas para a implantação do sistema. Inicialmente, foi realizado um diagnóstico da situação-problema verificando que em todo período de 21 anos de atividades, o acúmulo de prontuários ficou inoperante quanto ao acesso e guarda de documentos em papel.

As dificuldades do acesso por qualquer necessidade, em um total de papéis arquivados não permitia a contagem de sua numeração existente e apresentava dificuldade, por exemplo, no tempo que levava para encontrar uma determinada documentação. Em instância paralela, o envelhecimento dos prontuários provocava distúrbios à saúde dos profissionais do setor, exigindo redobrados cuidados aos mesmos.

A etapa seguinte foi a organização de prontuários médicos no hospital através do Serviço de Arquivo Médico (SAME), que atualmente possui duas salas. A sala principal com aproximadamente $89 \mathrm{~m}^{2}$, equipada com armários deslizantes e uma sala complementar de aproximadamente $35 \mathrm{~m}^{2}$, organizada com prateleiras de metal onde encontra-se também quantitativo de prontuários não catalogados.

A otimização do espaço físico e condução dos recursos humanos observou as fases essenciais para o início de todo processo de digitalização:

a) Equipe certa - o gerenciamento é liderado por um gerente administrativo, um supervisor que controla a classificação dos prontuários e a equipe de catalogadores e digitalizadores totalizando quatro profissionais no 
âmbito dos sistemas de controle;

b) Espaço físico - a área por pessoa é de $12 \mathrm{~m} 2$, considerando a preparação de arquivos e os equipamentos de digitalização - scanners, com velocidade de leitura e recursos de armazenamento necessários para cumprir o cronograma do projeto;

c) Software de digitalização adequado - Klinikos - é o sistema de gerenciamento eletrônico que concentra os primeiros módulos instalados com a entrada de dados que permite gerar os relatórios necessários ao movimento de prontuários de pacientes. São observadas as etapas de automação, indexação, imagem e identificação de dados;

d) Políticas e procedimentos claramente documentados - todas as instruções de entrada de dados, armazenamento, processamento e saídas seguem devidamente todas as instruções, inicialmente, abordadas e determinadas, seguindo as exigências do sistema instalado, para evitar ônus, imprecisões e inconsistências;

e) Acesso aos documentos digitalizados - a dinâmica de relatórios está estabelecida, principalmente, na confiabilidade, rapidez e eficiência diante das necessidades dos prontuários digitalizados;

f) Destino dos documentos digitalizados - considerando os benefícios da digitalização documental da maternidade são devidamente catalogados, digitalizados, armazenados em caixas de arquivo verde (o que preconiza o arquivamento na área da saúde), contabilizados em 30 prontuários por cada caixa, feito a identificação das caixas e enviados ao órgão controlador para o arquivo permanente, que é o arquivo da Secretaria de Saúde do Estado (SES).

O projeto prevê visitas da Administração superior com vistas ao reconhecimento do processo para determinar reuniões que possibilitam equacionar os problemas que eventualmente possam ocorrer. Toda equipe deve atender aos critérios determinados para consolidar toda elaboração com o resultado esperado diariamente, a fim de atender todo cronograma, não havendo ainda um período determinado para a finalização da digitalização dos prontuários elaborados manualmente e, consequentemente, dar continuidade ao processo eletrônico, quando atualizado, para atender com mais eficiência todo sistema documental do hospital.

A coleta dos dados deu-se a partir do acesso aos prontuários da Maternidade de Alta Complexidade do Maranhão-MACMA com autorização da Diretoria da unidade e da instituição gestora por Carta de Anuência.

\section{RESULTADOS}

Os resultados apresentados referem-se ao processo em andamento de digitalização dos prontuários das pacientes na MACMA, em que são notificadas as informações desde a admissão até o arquivamento definitivo.

\section{Estrutura da MACMA}

A Maternidade de Alta Complexidade do Maranhão (MACMA) está localizada à Av. Jerônimo de Albuquerque, $\mathrm{S} / \mathrm{N}$, bairro Cohab Anil III, na capital maranhense, São Luís, com 163 leitos, para atendimento a pacientes obstétricas, como referência à atenção à saúde na gestação de alto risco e ambientação adequada para o acolhimento e atendimento humanizado.

O hospital é uma unidade completa e oferece espaço para atendimento digno à população maranhense. A Unidade de Terapia Intensiva Materna (UTIM) da maternidade atende pacientes obsté- tricas graves, nos períodos pré, intra e pós-parto, com atendimento às doenças próprias da gravidez ou nela intercorrentes e que necessitem de internação em regime de cuidados intensivos.

A equipe de profissionais é formada por especialistas em terapia intensiva e ginecologistas obstetras, além de uma equipe de enfermagem especializada em UTI, assim como a presença e o apoio de médicos nas especialidades de cardiologia, anestesiologia, neurologia, infectologia, fisioterapia respiratória e psicologia, entre outras necessidades prementes, refletindo na redução dos indicadores de mortalidade materna.

Com a gestão do Instituto Acqua em parceria com a Secretaria de Estado da Saúde, sucederam-se mudanças na maternidade desde maio de 2015, como reorganização, ganho de novos leitos e equipamentos, o que reforça o papel de referência no atendimento às gestantes de alto risco em todo Estado.

SAME

O Serviço de Arquivo Médico (SAME) que opera dentro da maternidade tem por finalidade a guarda e a preservação do prontuário do paciente (dados pessoais, evolução clínica, exames, radiografias etc.) e a elaboração de relatórios e boletins estatísticos referentes ao movimento hospitalar. É um serviço imprescindível ao hospital, permitindo estimar o valor do trabalho profissional e o grau de eficiência com que são tratados os pacientes que recorrem ao mesmo.

O SAME funciona 24 horas para atender às demandas assistenciais da Instituição, manuseando diariamente cerca de 300 prontuários (arquivamento e desarquivamento), que estão disponíveis para consultas ambulatoriais, internações, pesquisa científica e solicitação da Direção. A equipe de trabalho do SAME é constituída por 13 funcionários efetivos que estão distribuídos em três turnos (manhã, tarde e plantão noturno). 
As atividades incluem:

a) Localizar e fornecer prontuários solicitados para consultas ambulatoriais, internação, estudos, pesquisa, auditoria;

b) Manter o controle de saídas e devolução de prontuários, fornecidos para consultas ambulatoriais, unidades de internação e/ou setores da maternidade;

c) Manter o registro numérico nos prontuários pelo método Dígito Terminal Simples com base no registro gerado pelo Sistema KLINIKOS no momento da abertura;

d) Receber, revisar e ordenar os prontuários de pacientes de alta, que retornam

do Faturamento;

e) Unificar os prontuários;

f) Manter sempre atualizada a documentação do prontuário do paciente;

g) Revisar periodicamente as seções de arquivamento, corrigindo eventuais falhas;

h) Selecionar prontuários para uso das comissões hospitalares;

i) Colaborar nas pesquisas científicas e nos trabalhos de investigação, quando solicitado;

j) Preparar os relatórios estatísticos mensais, trimestrais e anuais, referente às suas atividades, de interesse do Hospital;

k) Receber e providenciar respostas de informações aos interessados, quando autorizados pela Diretoria da Maternidade;

I) Capacitar os funcionários lotados no SAME.

O SAME se entrosa com os demais serviços técnicos e administrativos da Instituição, colaborando no aprimoramento da assistência prestada ao paciente. É o responsável por organização, armazenamento e guarda de prontuários dos pacientes, permitindo sua rastreabilidade sempre que necessário.

\section{Diagnóstico Situacional}

A MACMA foi fundada em1998 com o nome Hospital e Maternidade Marly Sarney, para atender às necessidades assistenciais da população ludovicense, atendendo, atualmente, usuários de outros municípios e distritos vinculados à cidade de São Luís.

O SAME dispõe, atualmente, de 1 Arquivo Médico Corrente, constituído por acervos de Prontuários Correntes e Intermediários, dispondo aproximadamente de 69,8 metros lineares de documentos arquivados, que datam de 1998 a 2019. A carência de espaço físico adequado para armazenamento e preservação dos prontuários, é evidente nesse Arquivo, considerando que o SAME não dispõe de espaço físico para ampliação do Acervo.

Assim, a importância do Arquivo para a instituição está ligada ao aumento expressivo do volume de documentos que utiliza no exercício de suas atividades e a necessidade de se estabelecerem critérios de guarda. A adoção de técnicas arquivísticas adequadas permite não apenas a localização eficiente da informação desejada, mas também a economia de recursos para a instituição.

Processo de digitalização dos prontuários

A partir da iniciativa de contratação pela SES-MA, a tecnologia KLINIKOS foi instalada junto ao departamento de

Quadro 1 - Relatório dos Prontuários Digitalizados. São Luis, MA, Brasil, jul/dez 2018

TOTAL MENSAL PRONTUARIOS DIGITALIZADOS

MÊS TOTAL

JULHO

AGOSTO 84

SETEMBRO 238

OUTUBRO 298

NOVEMBRO 263

DEZEMBRO 247

TOTAL
TI da maternidade com funcionalidade para os registros eletrônicos, incluindo o atendimento em todos os níveis assistenciais: clínico, administrativo, operacional e gerencial, significando registros quanto às internações, emergência, ambulatórios, farmácia, consultas, exames e prontuários médicos.

No módulo de documentação médica, o sistema controla a abertura e movimentação de prontuários permitindo a visualização do histórico assistencial e consulta dos resumos dos atendimentos. O sistema proporciona integração entre os registros de forma multidisciplinar.

Resultados do andamento do processo de digitalização

$\mathrm{O}$ arquivamento da documentação da maternidade deu-se a partir de 1998, marcando 21 anos de evolução do acervo de prontuários até o ano em curso. O início do processo de arquivo eletrônico foi a partir de julho de 2018. O montante de prontuários digitalizados até junho de 2019 somam 3.603 registros, nos quais foram trabalhadas as etapas de acordo com a realidade da unidade, e com apoio e conhecimento da Secretaria de Estado da Saúde, Instituto Acqua e o setor técnico de informática da maternidade. A evolução dos números é apresentada nos quadros e gráficos que seguem abaixo, referentes ao segundo semestre de 2018 e primeiro semestre de 2019: 


\section{Distribuição Percentual de Prontuários Digitalizados \\ - Mensal - $2^{\circ}$ Semestre 2018}

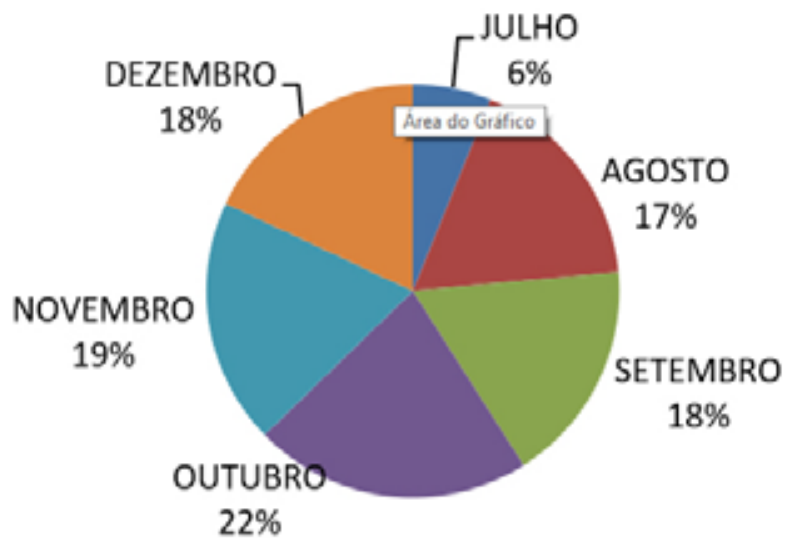

Quadro 2. Relatório de folhas de Prontuários Digitalizados. São Luis, MA, Brasil, jul/dez 2018 TOTAL MENSAL PRONTUARIOS DIGITALIZADOS

\begin{tabular}{lc} 
MÊS & TOTAL \\
\hline JULHO & 4526 \\
\hline AGOSTO & 11.532 \\
\hline SETEMBRO & 11.481 \\
\hline OUTUBRO & 15.780 \\
\hline NOVEMBRO & 13.716 \\
\hline DEZEMBRO & 12.558 \\
\hline TOTAL & 69.593
\end{tabular}

Observa-se no Quadro 1, o montante inicial de 84 prontuários digitalizados em julho de 2018, representando 4526 folhas. A evolução gradativa se deu acompanhada pelo supervisor dos trabalhos, seguindo em agosto do período semestral, com $17 \%$, inseridos no modulo de prontuários, Gráfico 1, e folhas respectivas, Gráfico 2. Relacionados aos meses seguintes, em dezembro/2018 o total de prontuários digitalizados foi 1.369, Quadro 1 , sendo o número de folhas fechado em 69.593, Quadro 2.

Gráfico 2. Percentuais de folhas de Prontuários Digitalizados. São Luis, MA, Brasil, 2018

\section{Distribuição Percentual de Folhas Digitalizadas} $2^{\circ}$ Semestre 2018

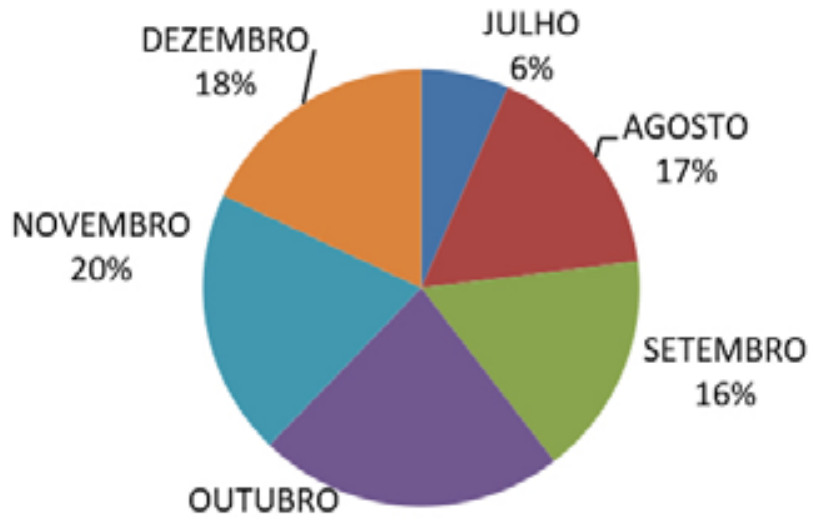


Quadro 3 - Relatório dos Prontuários Digitalizados. São Luis, MA, Brasil, jan/ jun 2019

\section{TOTAL MENSAL PRONTUARIOS DIGITALIZADOS}

MÊS TOTAL

JANEIRO 399

FEVEREIRO 378 MARÇO 314

ABRIL 381 MAIO 444

JUNHO

318

TOTAL

2.234

Gráfico 3. Percentuais de Prontuários Digitalizados. São Luis, MA, Brasil, 2019

\section{Distribuição Percentual de Prontuários Digitalizados Mensal - $1^{\circ}$ Semetre 2019}



Quadro 4. Relatório de folhas de Prontuários Digitalizados. São Luis, MA, Brasil, jan/jun 2019

\section{TOTAL MENSAL PRONTUARIOS DIGITALIZADOS}

MÊS

TOTAL

JANEIRO

19.204

FEVEREIRO

16.340

MARÇO

14.841

ABRIL

18.076

MAIO

20.908

JUNHO 17.950

TOTAL
No período de janeiro a junho de 2019, o número de prontuários digitalizados correspondeu a 2.234, somados ao segundo semestre de 2018, fecha o montante apresentado de 3.603 registros com 176.973 folhas. A variação percentual diária e mensal não é estável devido algumas circunstâncias consideradas na preparação da documentação, a média diária de inserção no modulo pode ser considerado entre 21 a 23 prontuários.

\section{DISCUSSÃO}

A importância que se dá nesse processo é o fato de que o prontuário do paciente se constitui o documento que inclui e relata toda história e vida clínica do mesmo. Nesse aspecto, tem valor imensurável para a instituição, assim como para o próprio paciente e para a equipe dos profissionais que forma a instituição hospitalar. Portanto, deve receber um tratamento diferenciado, ser bem elaborado e manuseado com cuidado, zelando-se pela integridade dos seus componentes. É um instrumento legal que comprova o atendimento prestado ao paciente e possibilita uma análise acurada dos fatos ocorridos, servindo de instrumento de defesa ou acusação, dada a sua elaboração. Assim, a sua guarda deve atender aos critérios estabelecidos.

Remete-se ainda à confiabilidade das informações, a segurança e o sigilo das mesmas, que devem ser observadas segundo os padrões exigidos, especificados e disponibilizados pela Resolução CFM n. ${ }^{\circ} 1.821 / 2007$, que inclusive pode ser submetido a uma comissão especial para conceder o selo de qualidade relacionado com os sistemas informatizados. As exigências culminam também no prazo mínimo de vinte anos, a partir do último registro, para a preservação do prontuário físico de paciente que não foi arquivado digitalmente. A digitalização dos prontuários concorre para ganho de tempo nas buscas de informações do paciente, facilita o manuseio dessas, diminuído o 
Gráfico 4- Percentuais de folhas de Prontuários Digitalizados. São Luis, MA, Brasil, jan/jun 2019Fluxograma de seleção dos artigos. Porto Alegre, RS, Brasil, 2019

\section{Distribuição Percentual de Folhas Digitalizadas - Mensal - $1^{\circ}$ Semestre 2019}

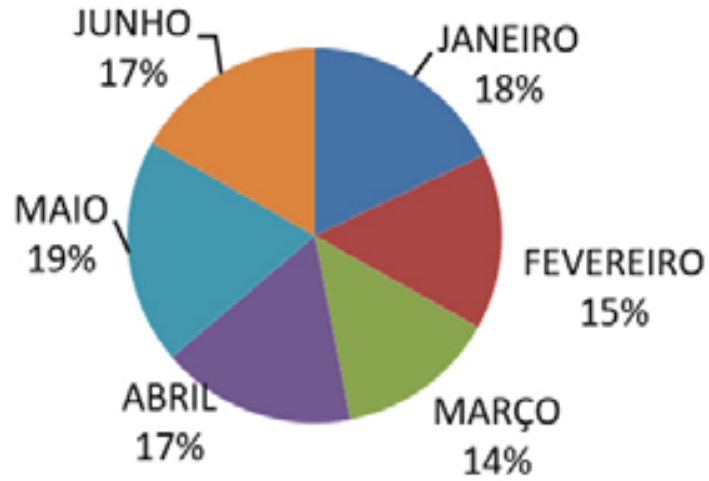

espaço físico para sua preservação.

Os trabalhos realizados na MACMA, conjuntura deste estudo, notifica a importância da preservação de informações necessárias e imprescindíveis na vivência de atendimentos à pacientes obstétricas com suas inúmeras necessidades, muitas vezes de risco. Eleva-se, portanto, o grau de relevância quanto ao uso das tecnologias de armazenamento dos dados na instituição.

Assim, com o processo em pleno funcionamento na MACMA, o que vem ocorrendo há um ano, é possível mensurar os principais ganhos proporcionados pela iniciativa, entre os quais merece destaque a agilidade na procura, visualização e impressão dos prontuários pelos profissionais do setor, considerando a necessidade de aprimorar o espaço físico. De todo período de digitalização de prontuários na referida unidade de saúde, os dados referentes a 11 anos já estão sendo catalogados e arquivados adequadamente para entregar à Secretaria de Estado de Saúde para o arquivo permanente. O volume de prontuários digitalizados até o momento presente altera-se diariamente dando origem a um novo cenário de arquivo, eficiente, seguro e rápido.

\section{CONCLUSÃO}

Devido aos inúmeros benefícios que o investimento tecnológico em prontuário eletrônico poderá trazer para a unidade hospitalar MACMA, o uso de software atende melhor às demandas da instituição, representando a expansão no atendimento à saúde de seus pacientes.

A maternidade, em parceira com o Instituto Acqua, vem, nos últimos tempos, investindo neste projeto de prontuários, e o uso de tais tecnologias se constitui em facilitarmos trabalhos que se realizam, levando a concluir ser este projeto a construção de um marco de maior referência e eficiência.

A preocupação inicial de como preservar a documentação de registro do atendimento aos pacientes, ponto relevante nesse projeto, encontrou solução para o controle do volume de prontuários que se forma desde a inauguração das atividades da maternidade, no processo de digitalização dos prontuários.

O projeto apresenta-se como embrionário, porém, a documentação digital da Maternidade de Alta Complexidade do Maranhão conduz a uma melhor condição de andamento administrativo. Essa referência, certamente, estende-se a outras unidades de atendimento à saúde, como, por exemplo, as demais unidades sob a gestão do próprio Instituto Acqua, com tecnologias que refletem maior eficiência.

\section{Referências}

1. Teófilo RB, Freitas LS. $O$ uso de tecnologia da informação como ferramenta de gestão. Gestão de Negócios pela FGV, Universidade Federal de Campina Grande, SEGET [Internet]. 2019 [acesso em 08 mai 2019].

Disponivel em: https://www.aedb.br/seget/arquivos/artigos07/652_ SEGET\%20roro.pdf.

2. Souza P, Petri SM. As Tecnologias da Informação no Processo de Gerenciamento: Avanços do Governo Eletrônico. IX Simpósio de Excelência em Gestão e Tecnologia [Internet]. 2012 [acesso em 10 mai 2019]. Disponível em https:// www.aedb.br/seget/arquivos/artigos12/45016472.pdf.

3. Mai S, et al. 0 Uso das Tecnologias na Democratização da Informação em Saúde. RGSS. 2017 set./dez.; 6(3).

4. Silva TCF. Gestão de Documentos em Arquivo Hospitalar. 2008. Monografia (Curso de Graduação em Biblioteconomia). Universidade Federal do Rio Grande do Norte [Internet]. 2016 [acesso em 08 mai 2019]. Disponivel em: https:// simagestao.com.br/wp-content/uploads/2016/01/TeresaCFS_Monografia.pdf. 5. Pinto VB. Prontuário eletrônico do paciente: documento técnico e informa- ção e comunicação do domínio da saúde. Enc. Bibli.:R. Eletr. Bibliotecon. Ci. Inf., Florianópolis, n. 21, $1^{\circ}$ sem. 2006.

6. Pazin M. Arquivos de empresas: tipologia documental. São Paulo: Associação de Arquivistas de São Paulo; 2005.

7. Ministério da Fazenda, Secretaria Executiva, Subsecretaria de Planejamento, Orçamento e Administração, Coordenação-Geral de Recursos Logísticos (BR). Procedimentos para digitalização de documentos e processos: cartilha. Brasília: MF/SE/SPOA/COGRL, 2017.

8. Ministério da Saúde (BR). GM. Portaria n. ${ }^{\circ}$ 900, de 31 de março de 2017. Dispõe sobre a implantação e o funcionamento do Sistema Eletrônico de Informações-SEl [Internet]. 2017 [acesso em 19 jul 2019]. Disponível em: http:// bvsms.saude.gov.br/bvs/saudelegis/gm/2017/prt0900_310_03_2017.html. 9. Conselho Federal de Medicina. Resolução n. ${ }^{\circ} 1.821 / 2007$ [Internet]. Publicada no D.O.U. de 23 nov. 2007, Seção l, pg.252 [acesso em 19 jul 2019]. Disponível em: https://sistemas.cfm.org.br/normas/visualizar/resolucoes/ BR/2007/1821. 